\title{
Frei ausgewählte Diät wirkt nicht besser
}

\author{
Eine Studie wollte ermitteln, um wie viel besser die Gewichtsreduktion funktioniert, wenn \\ ältere Patienten die Nahrungszusammensetzung beim Abspecken selbst bestimmen können. \\ Das Ergebnis: Die freie Auswahl brachte gar keinen Vorteil.
}

Für die Studie wurden Patienten mit einem BMI > 30 und einem Alter ab 75 Jahren in zwei Gruppen randomisiert. In einer durfte man sich die Diätform eigenständig aussuchen, in der anderen wurde sie durch ein Randomisierungsprotokoll festgelegt.

In der ersten Gruppe wurde zunächst mittels Fragebogen (FPQ) die Nahrungspräferenz erhoben. Anschließend konnten sich die 105 Probanden zwischen zwei Diäten entscheiden. 58\% wählten eine kohlenhydratarme (LCD), $42 \%$ eine fettreduzierte Diät (LFD). Nach 12 Wochen durfte gewechselt werden, was 5 Probanden auch taten. Von den 102 Teilnehmern mit vorgegebener Gruppeneinteilung wurden 52\% in die LCD-Gruppe und $48 \%$ in die LFDGruppe randomisiert. Die Intervention erfolgte über 48 Wochen. In der ersten Hälfte gab es alle zwei Wochen, danach monatlich Gruppenschulungen. Zwischendurch berieten Ernährungsberater die Teilnehmer telefonisch.

Der adjustierte Gewichtsverlust in der Gruppe mit freier Diätwahl betrug nach 48 Wochen 5,7 kg (5,6\%), während er in der Gruppe mit fester Randomisierung zu den unterschiedlichen Diätformen $6,7 \mathrm{~kg}(6,5 \%)$ betrug. Hier ergab sich kein statistisch signifikanter Unterschied $(\mathrm{p}=0,26)$.

Auch in der Untersuchung sekundärer Outcome-Parameter wie Diätadhärenz, körperlicher Aktivität und Lebensqualität gab es keinen Unterschied zwischen den Gruppen. In einer explorativen Analyse fand sich bei den Patienten, die mehr als 15 Gruppensitzungen absolviert hatten, ein numerisch höherer Gewichtsverlust (7,6 vs. 2,7 kg in der Auswahlgruppe und 8,2 vs. $2,8 \mathrm{~kg}$ in der fixen Randomisierungsgruppe).
- Yancy WS Jr, Mayer SB, Coffman CJ et al. Effect of Allowing Choice of Diet on Weight Loss: A Randomized Trial. Ann Intern Med. 2015;162:805-14

\section{KOMMENTAR}

Auch wenn die Probanden älter und zu 73\% männlich waren, passen die Daten gut ins Bild früherer Interventionen. Sie stützen die Annahme, dass die freie Auswahl einer Ernährungsform eher nicht von Vorteil ist, ja ggf. sogar einen geringen Nachteil mit sich bringt. Auffällig ist die Tatsache, dass die Teilnahme an den Gruppensitzungen ein wesentlicher Prädiktor des Gewichtsverlustes ist. Es lässt sich nur vermuten, inwieweit hier striktere Interventionsregime oder die Gruppendynamik ausschlaggebend sind. Motivationsstrategien mit Gruppensitzungen scheinen aber erfolgversprechend zu sein.

PD Dr. med. K. Mai

\footnotetext{
$\rightarrow$ Um effiziente Abspeck-Methoden geht es auch im Report ab S. 8.
}

\section{Die Mundhöhle gehört zum Magen-Darm-Trakt}

Ein 28-jähriger Mann bekam seit zwei Jahren immer wieder einmal spon$\tan$ auftretende Hautulzerationen, aus denen sich Sekret entleerte (Abb. A und B). Sechs Jahre zuvor war bei ihm ein Morbus Crohn diagnostiziert worden, der mit Methotrexat und Adalimumab behandelt wurde. Wegen des Auftretens von Fisteln im Bereich von Dünn- und Dickdarm war er mehrfach operiert worden und hatte nun eine lleostomie sowie einen Rek-

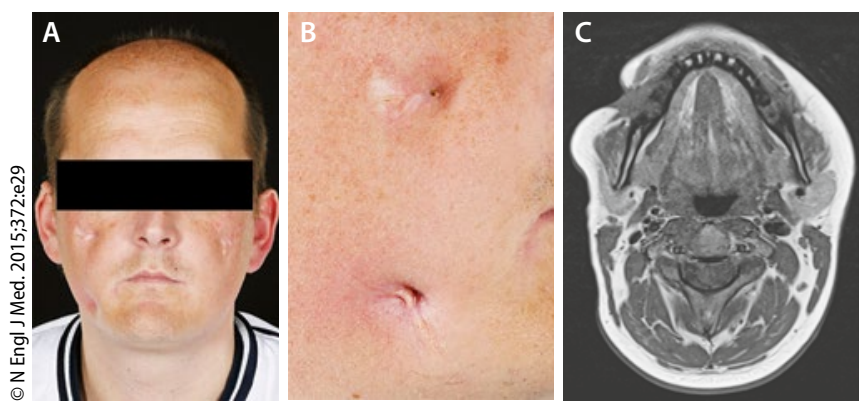

Hautulzerationen im Gesicht (A, B); von Ulzera in der Mundhöhle ausgehende orokutane Fisteln (C). tumstumpf. Die MRT-Bildgebung der orofazialen Region (Abb. C) und eine Kontrastmitteldarstellung ergaben drei orokutane Fisteln, die von Ulzera in der Mundhöhle ausgingen und auch eine Verbindung zu Zahntaschen hatten. Bei der Extraktion eines Zahnes und der Kürettage der Fistelhöhlen fand man histologisch entzündliches Granulationsgewebe ohne Erregernachweis.

Beim Morbus Crohn handelt es sich um eine chronische granulomatöse Entzündung, die jeden Teil des Gastrointestinaltrakts vom Mund bis zum Anus treffen kann. Orofaziale Beteiligungen sind zwar selten, aber besonders unangenehm für den Betroffenen. Obwohl kein Erreger nachgewiesen werden konnte, verschlossen sich die Fisteln nach Zahnextraktion und einer antibiotischen Behandlung. Bei einer Nachuntersuchung ein Jahr später war es im Gesichtsbereich nicht zu einem Rezidiv gekommen. Die tief eingezogenen Narben wird der Patient behalten. Sie geben auch eine Vorstellung davon, wie leicht eine derart intensive Narbenbildung im Bereich des Dünndarms zu fatalen Stenosen führen kann.

Prof. Dr. med. H. S. FüeßI

- Goel RM, Hullah E (rgoel50@hotmail.com). Orofacial fistulae associated with Crohn's disease. NEngl J Med. 2015;372:e29 